\title{
Effect of Intermittent Pneumatic Compression (IPC) on Glucose Regulation and Inflammation in Type 2 Diabetes
}

\author{
Caitlin S Nagy ${ }^{2}$, Kenneth Sparks ${ }^{1 *}$, Emily Kullman ${ }^{3}$, Douglas Wajda ${ }^{4}$ and Shana Williams ${ }^{5}$ \\ ${ }^{1}$ Exercise Physiology, Cleveland State University, USA \\ ${ }^{2}$ Exercise Science, Cleveland State University, USA
}

${ }^{3}$ Human Bioenergetics, Cleveland State University, USA

${ }^{4}$ Kinesiology, Cleveland State University, USA

${ }^{5}$ Northeast Ohio Medical School, USA

*Corresponding author: Kenneth Sparks, PhD Exercise Physiology, Cleveland State University

2121 Euclid Ave PEB60 Cleveland, Ohio 44115, USA.

To Cite This Article: Kenneth Sparks. Effect of Intermittent Pneumatic Compression (IPC) on Glucose Regulation and Inflammation in Type 2 Diabetes. Am J Biomed Sci \& Res. 2019 - 5(6). AJBSR.MS.ID.000963. DOI: 10.34297/AJBSR.2019.05.000963.

Received: 製September 28, 2019; Published: 掣 October 17, 2019

\begin{abstract}
Purpose: The purpose of this study is to determine whether the intermittent pneumatic compression (IPC) device improves glucose regulation and inflammation in participants with type 2 diabetes. It was hypothesized that the intermittent pneumatic compression device will have positive effects on glucose regulation and inflammation in individuals with type 2 diabetes.
\end{abstract}

Methods: Subjects included 10 individuals diagnosed with type 2 diabetes. Baseline measures of lipid profile and HbA1C were obtained by a finger stick. Plasma and serum were obtained intravenously to analyze insulin, glucagon, Interleukin 6 (IL-6) and C-reactive protein (CRP). The subjects came in a total of 3 times to the laboratory to be measured for the variables previously listed. The subjects completed a total of 35, IPC treatments (5 days/week for 7 weeks) by using the Norma Tec recovery system (NT).

Results: No significant differences found within lipid profile or inflammation. CRP values decreased from the $1^{\text {st }}$ to the $3^{\text {rd }}$ treatment, but no significant difference was found. HbA1C was found significant $(p=.002, p=.026)$ from $1^{\text {st }}$ to $2^{\text {nd }}$ and $1^{\text {st }}$ to $3^{\text {rd }}$ treatments.

Conclusion: Previous research has shown an increase in blood flow from the use of the IPC devices. An increase in blood flow to the lower extremities can lead to positive effects in endothelial function, decreased inflammation and with further research, a decrease in HbA1C and other glucose regulators.

\section{Introduction}

Diabetes is a disease that has become prevalent among adults and children. Raaijmakers, et al. [1] discussed how diabetes is a chronic illness that requires intense, and constant medical care. The varying types of diabetes, type 1 and type 2 require different forms of care for an individual dealing with this disease. Type 1 can be developed from birth or at an early age and is brought on by the individual not being able to produce insulin to maintain one's blood sugar levels. Type 2 can develop over time from several factors such as, diet or other preexisting health issues. Type 2 is brought on by the individual being insulin resistant, causing the insulin being produced to be non-responsive by the body. Insulin is an important factor when dealing with type 1 and type 2 diabetes. Insulin is a hormone produced by the pancreas that lowers glucose levels within the blood. When glucose levels rise, insulin is secreted by the beta cells of the islets of Langerhans inside the pancreas Wilcox [2]. If this process is disrupted, glucose will not be able to be taken into the cells and used for energy. Therefore, causes insulin resistance and an increase in glucose within the blood which can have harmful effects on the body. Monitoring insulin levels is very important in individuals with diabetes. High amounts of cholesterol in the blood and hypertension can also be caused from type 2 diabetes because of the difficulty in metabolizing glucose. If uncontrolled diabetes progresses, other cardiovascular risks may develop over time. 
Glucagon is a hormone produced by the pancreas that secretes when blood glucose levels fall too low. For individuals with type 2 diabetes, it is shown that glucagon raises blood glucose levels too high because of the impaired recognition of insulin. Ferrannini, et al. [3] researched insulin resistance by the hyperinsulinaemic clamp in 1,296 non-diabetic subjects. The researchers found that whole body insulin resistance shows an association with increased fasting glucagon levels, as a possible result of alpha cell insulin resistance. Increased levels of glucagon in the body may influence insulin resistance in type 2 diabetics.

Enhanced external counter pulsation (EECP) is a method that has been used to treat patients with coronary artery disease. EECP is very similar to IPC because of the method of using compression to help treat health conditions. This method is noninvasive and uses cuffs that inflate and deflate to increase venous return to the heart and decrease left ventricular afterload Lawson, et al. [4]. Levenson [5] researched cyclic GMP (cGMP) release by using acute enhanced external counter pulsation. EECP is typically used to help treat individuals with CAD. Arterial function is related to the release of cGMP and regulates vascular smooth muscle and affects growth of cells and division. This study had a one-time treatment of EECP to examine the effects of cGMP levels. Significance was determined at $\mathrm{p}<.05$. Results of the study showed a significant increase in cGMP for plasma concentration $(\mathrm{p}<.001)$ and platelet content $(\mathrm{p}<.01)$. The EECP showed positive effects with increasing cGMP in patients with CAD and cardiovascular risks.

Feldman et al. [6] examined EECP on patients experiencing chronic heart failure. EECP has been known to decrease the amount of chest pain and extends time to exercise induced ischemia in patients with coronary artery disease and angina. There were 187 participants within the study, the subjects were randomized within a 1:1 ratio to EECP treatment or continued physical therapy. Sessions lasted 1 hour and were completed 35 times over 7-8 weeks. The pneumatic cuffs were placed specifically around each lower extremity and the buttocks. The pressure of the cuffs was pumped up to the onset of diastole and were released before the onset of systole. Pressure was set to $300 \mathrm{mmHg}$ and was reached within the first five minutes of the beginning of the start of treatment Pulse oximetry and clinical status was monitored throughout the testing. Both groups exercised, but the group that received EECP treatment increased 60 seconds more when exercise was tested after 7-8 weeks. This study suggests that 35, 1-hour treatment of EECP will have a positive effect and benefit patients with heart failure and systolic left ventricular dysfunction.

Inflammation is very common amongst individuals with type 2 diabetes. High blood glucose levels can play a role in inflammation in the blood vessels and can cause other diabetic complications. The connection between type 2 diabetes and inflammation is still being researched, but the increased fat tissue seems to have a small connection with inflammation and insulin resistance. C-reactive protein (CRP) and interleukin 6 (IL-6) are the most common markers to test for inflammation within the blood Singh-Manoux, et al. [7]. An increase in systemic inflammation can increase an individual's risk for cardiovascular diseases and insulin resistance Haffner [8]. Insulin resistance can be caused by chronic inflammation among individuals that have type 2 diabetes. Insulin resistance is the body's way of rejecting the insulin that is being produced, which induces blood sugar to rise. Being able to understand how sensitive the body reacts to insulin is important when trying to control or prevent the onset of diabetes.

Intermittent Pneumatic Compression (IPC) is a device used by inflating and deflating varying types of cuffs around the limbs to improve venous circulation in individuals suffering from diabetes, edema, thrombosis, cardiovascular risks, etc. This type of compression treatment has also been used to treat swelling after a traumatic incident and possibly after surgical procedures Almstedt and Lewis, [9]. Martin and Braith [10] found significant evidence showing decreases among high sensitivity C-reactive protein while using an EECP device, which has similar effects to an IPC device. Seeing decreases in any compression treatments shows promising evidence that this could be a big step towards controlling and preventing any future cardiovascular risks for type 2 diabetes. IPC has been used in various research studies to help aid athletes in decreasing inflammation and speed up the recovery process. It has been used frequently in the field of sports medicine for the treatment of small injuries Sutkowska [11].

Taking all the necessary measures to try to prevent or control one's diabetes can reduce the risk of other medical complications such as, kidney failure, cardiovascular issues and hypertension Raaijmakers, et al. [1]. Sheldon, Roseguini, Laughlin, Newcomer [12] discussed the effective point that makes this compression method so easy is that it can be done at the individual's home. Studies that have researched IPC have found that this method elevates blood flow, reduces risks of negative vascularity and helps with relieving ischemia.

External compression shows changes in the hemodynamics and the structure of individuals suffering from lower extremity conditions. This compression method applies intense pressure that encloses the lumen, causing venous emptying and preventing stasis Chen, Frangos, Kilaru and Sumpio [13]. Swelling is caused by the increase in pressure on the extremities and puts $20 \%$ more tension on the endothelial cells. The pressurized blood moves through the veins and if the pressure is applied periodically then this could increase peak velocity of blood flow by $200 \%$ within the lumen Chen, Frangos, Kilaru and Sumpio [13]. Blood is drained at the site of compression, which provides a pulsatile flow through the compressed extremity.

A systematic review was done over IPC for individuals with critical limb ischemia. There were three different types of IPC that were researched in this review, such as Art Assist device, Arterial 
Flow device, and another compression device that is involved with ventricular contraction of the heart Moran, Teljeur, Harrington and Ryan [14]. IPC is supported to have positive effects and prevent further risks for individuals with critical limb ischemia Moran, Teljeur, Harrington and Ryan [14]. This systemic review showed inconsistent research with IPC treatment and individuals with critical limb ischemia who aren't suitable for percutaneous transluminal angioplasty or surgical revascularization.

A similar study, Karvos, et al. [15] discussed intermittent pneumatic compression and its effects on a controlled study with an 18th month follow up for individuals with critical limb ischemia. The purpose of this study was to evaluate IPC in patients with critical limb ischemia, non-healing wounds, amputations and tissue loss. There were two groups within the study; group 1 consisted of 24 patients with a median age of 70 that used the IPC treatment to care for tissue loss and wound healing, group 2 consisted of 24 patients that did not use the treatment of IPC to help with the care of tissue loss and the healing of wounds produced by the critical limb ischemia Researchers found that wound healing and limb salvage was improved by the use of IPC treatment in group $1(\mathrm{p}<.01$, with a $p$ value of <.05). The treatment group showed significant increases in healing than the control group.

Venus leg ulcers are a common affliction that may affect individuals throughout their lifetime. A venous ulcer may be caused by either an injury on the lower extremity, poor circulation, obstruction of the vein lumen and incompetence of the valve (Comerota [16]. This condition can damage blood cells and be very painful. Venous leg ulcers cost the healthcare system a lot of money for treatment options. IPC has been researched in treatment/ prevention of venous leg ulcers among individuals. Articles on IPC and venous ulcers were reviewed, and the results have shown positive effects on venous ulcers by using the IPC treatment.

The purpose of this study is to determine whether the intermittent pneumatic compression (IPC) device improves glucose regulation and inflammation in participants with type 2 diabetes. It was hypothesized through extended research that intermittent pneumatic compression will have positive effects on glucose regulation and inflammation in individuals with type 2 diabetes.

\section{Methods}

This study included ten subjects, with an age range between 30-70 years that have been diagnosed with type 2 diabetes. The subjects were included having met inclusion criteria of type 2 diabetes. The participants involved within the study were recruited by word of mouth among the Cleveland community and throughout the Cleveland area. Each of the participants signed an IRB approved informed consent specifying all the procedures, potential risks and benefits.

Two weeks prior to the study, the subjects maintained a normal diet and physical activity pattern. The subjects came in fasted 10-
12 hours prior to arriving in the laboratory. The subjects came in a total of 3 times during the study for pre, mid and post testing during which plasma and serum blood samples were obtained. The morning of testing, baseline measures of serum, plasma, body composition, lipid profile and $\mathrm{HbA} 1 \mathrm{C}$ were obtained. Lipid profile, $\mathrm{HbA} 1 \mathrm{C}$ and glucose were obtained by a finger stick and analyzed in the Cholestech LDX. The blood received intravenously from the antecubital space was spun through a centrifuge to extract the isolated blood plasma and serum, which was pipetted into tubes and frozen until further analyzed. The pre, middle and post-plasma samples, were analyzed for C-reactive protein (CRP) Ray Biotech), Interleukin 6 (IL-6) (R\&D Systems), glucagon (Invitrogen) and insulin (Crystal Chem) using commercially available ELISA kits. Quality of life was measured pre-and post-intervention by using the SF-36 health survey.

The subjects participated in a 1 hour, at home, pneumatic compression treatment for 5 days a week for 7 weeks using the NormaTec recovery system. This accrued thirty-five treatments total for each participant during the study. The NormaTec recovery system is a large boot that fits around each leg of the participant and is divided into sections that are called cells. Each cell is around a part of the leg, the first cell (cell 1) is located on the foot and the cells continue up the leg until it reaches the top of the thigh (cell 5). Each subject received treatment at the same intensity setting of 10 (90 mmHg for cells 1 and 5 and $100 \mathrm{mmHg}$ for cells 2, 3 and 4) for one hour. Each cell compression has a duration of 30 seconds. During the 30 second compression and duration of the cells, there are 3 techniques, pulsing, gradients and distal release. Each part of these techniques is important for the movement of fluid, forced pressure, and circulatory flow within the muscles of the lower extremities. The recommendation for the intensity setting is to be at 10 (settings from 1-10) for the best results.

\begin{tabular}{|c|c|}
\hline & $\begin{array}{l}\text { - Informed consent, SF-36 } \\
\text { - Fasted } \\
\text { - Blood samples (plasma/serum), lipid profile, glucose, HbA1 }\end{array}$ \\
\hline $\begin{array}{l}17^{\text {th }} \\
\text { Assessment }\end{array}$ & $\begin{array}{l}\text { - Fasted } \\
\text { - Blood samples (plasma/serum), lipid profile, glucose, HbA } 1\end{array}$ \\
\hline & $\begin{array}{l}\text { - Fasted } \\
\text { - Blood samples (plasma/serum), lipid profile, glucose, HbA } \\
\text { - SF-36 }\end{array}$ \\
\hline
\end{tabular}

Subjects arrived halfway through the 7 weeks, arrived fasted (10-12 hours), lipid profile, glucose, HbA1C and venous blood draws were obtained, spun through the centrifuge and frozen to be further analyzed. During the 7th week, the participants came in a final time. They brought the device with them to return to the lab. Lipid profile, glucose and $\mathrm{HbA1C}$ were obtained and analyzed. Venous blood samples were obtained. The blood was spun in a centrifuge and frozen to be further analyzed. The presence of the CRP, IL-6, glucagon and insulin were analyzed by using the ELISA 
kit. SPSS version 22 was used for data analysis. For the conclusion of the study, the subjects were compensated for their participation in the study (Figure 1-4).
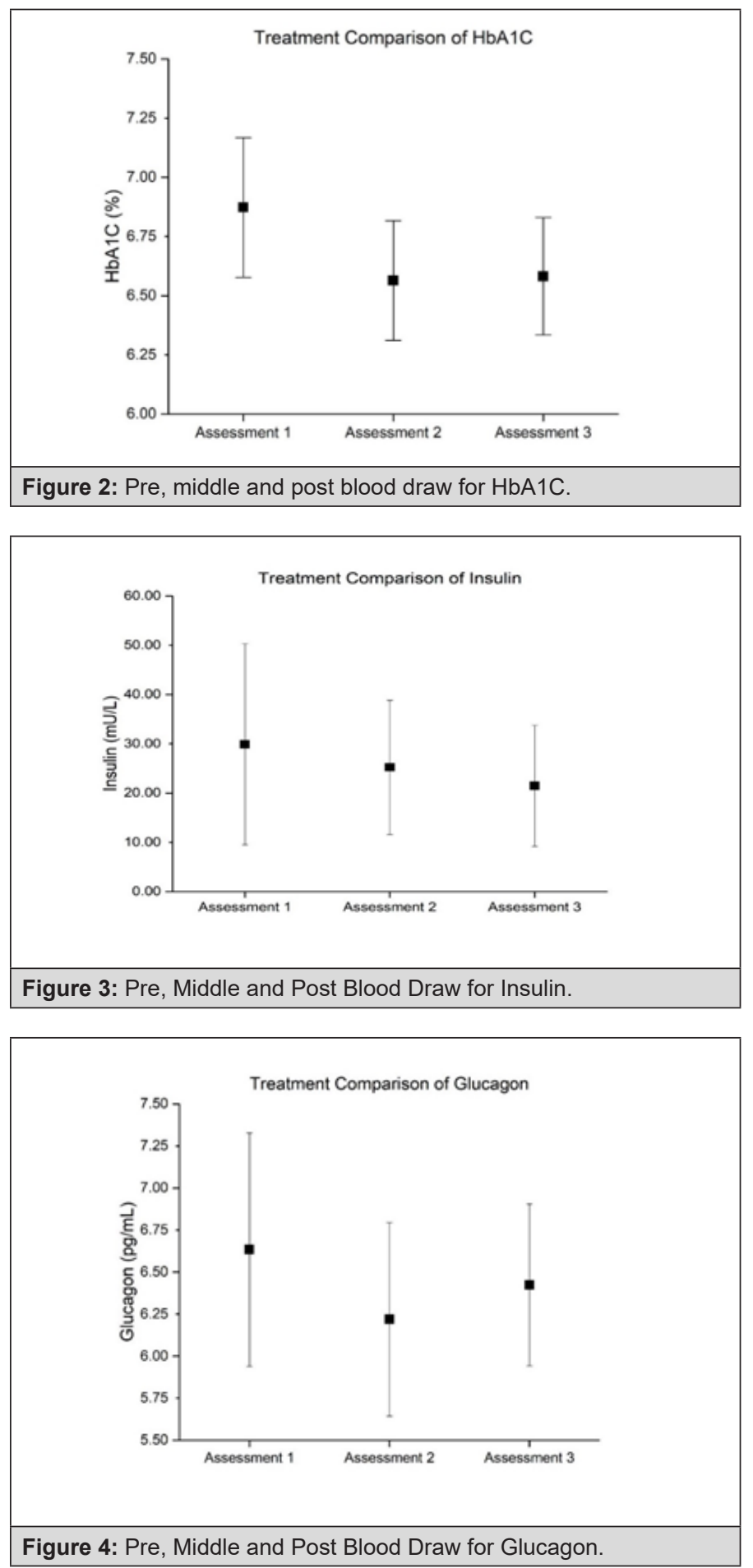

\section{Results}

Ten subjects with type 2 diabetes participated in the study to help determine whether glucose regulation and inflammation are positively affected by the NormaTec recovery system. The subjects performed 35 total pneumatic compression treatments for 1 hour a day, 5 days a week for 7 weeks. Blood measures were taken for the $1^{\text {st }}, 17^{\text {th }}$ and $35^{\text {th }}$ treatment of lipid profile and HbA1C, which were obtained by a finger stick. Plasma and serum were obtained $1^{\text {st }}$, $17^{\text {th }}$ and $35^{\text {th }}$ treatment intravenously to analyze insulin, glucagon, Interleukin 6 (IL-6) and C-reactive protein (CRP). Repeated measures ANOVA compared the lipid profiles, glucose regulation and inflammation markers during the 3 laboratory visits. The SF-36 health status questionnaire was compared pre and post intervention and analyzed using the repeated measures paired sample t-test. The graphs shown show +/- the standard error for each of the outcomes.

\section{Lipid Profile}

Results from the repeated measures ANOVA showed no main effect of time point on total cholesterol (TC), HDL, LDL or triglycerides (TRG). However, there was a slight decrease in mean values for total cholesterol $\left(1^{\text {st }}-35^{\text {th }}, 17^{\text {th }}-35^{\text {th }}\right)$, HDL $\left(1^{\text {st }}-17^{\text {th }}, 1^{\text {st }}-\right.$ $\left.35^{\text {th }}, 17^{\text {th }}-35^{\text {th }}\right)$, and LDL $\left(1^{\text {st }}-17^{\text {th }}, 1^{\text {st }}-35^{\text {th }}, 17^{\text {th }}-35^{\text {th }}\right)$ throughout the $1^{\text {st }}$ through the $35^{\text {th }}$ treatment blood measurement (Table 1 ).

\begin{tabular}{|c|c|c|}
\hline Variable & Mean \pm SD & Effect Size \\
\hline TC1 & $158.6 \pm 43.2$ & 0.089 \\
\hline TC2 & $162.0 \pm 56.5$ & \\
\hline TC3 & $153.2 \pm 39.8$ & \\
\hline HDL1 & $49.3 \pm 13.9$ & 0.124 \\
\hline HDL2 & $48.7 \pm 11.8$ & \\
\hline HDL3 & $46.7 \pm 10.5$ & \\
\hline TRG1 & $142.0 \pm 96.2$ & 0.097 \\
\hline TRG2 & $171.0 \pm 177.5$ & \\
\hline TRG3 & $181.9 \pm 185.9$ & \\
\hline LDL1 & $74.8 \pm 23.5$ & 0.133 \\
\hline LDL2 & $73.6 \pm 23.2$ & \\
\hline LDL3 & $68.3 \pm 25.8$ & \\
\hline
\end{tabular}

\section{Glucose Regulation}

Table 2: Comparison of Glucose Regulation Between the Pre, Middle and Post.

\begin{tabular}{|c|c|c|}
\hline Variable & Mean \pm SD & Effect Size \\
\hline HbA1C1 & $6.9 \pm 1.01$ & 0.467 \\
\hline HbA1C2 & $6.5 \pm .88^{*}$ & \\
\hline HbA1C3 & $6.5 \pm .86^{*}$ & \\
\hline INSULIN1 & $29.0 \pm 64.5$ & 0.08 \\
\hline INSULIN2 & $25.2 \pm 38.9$ & \\
\hline INSULIN3 & $21.5 \pm 38.7$ & \\
\hline GLUCAGON1 & $6.6 \pm 2.2$ & 0.033 \\
\hline GLUCAGON2 & $6.2 \pm 1.8$ & \\
\hline GLUCAGON3 & $6.4 \pm 1.5$ & \\
\hline GLU1 & $136.0 \pm 29.3$ & 0.073 \\
\hline GLU2 & $128.1 \pm 29.4$ & \\
\hline GLU3 & $137.7 \pm 34.6$ & \\
\hline
\end{tabular}


Results from the repeated measures ANOVA showed main effect of time point on HbA1C $(p=.015)$ in (Table 2) .Post-hoc comparisons showed that HbA1Cs at time points for the $17^{\text {th }}(p=$ .002 and $35^{\text {th }}(p=.026)$ treatment were significantly lower than time point 1 . Mean insulin values showed a slight decrease from the pre $\left(1^{\text {st }}\right)$ to middle $\left(17^{\text {th }}\right)$ treatment blood draw and the pre $\left(1^{\text {st }}\right)$ to post $\left(35^{\text {th }}\right)$ treatment blood draw, although no significance was found. The slight decrease shown in the other glucose regulators may have an influence on the significant decrease in HbA1C.

\section{HbA1C}

The analysis shows a significant decrease in HbA1C among the three treatment blood draws throughout the 7 weeks. There was a significant difference in treatments pre and middle $\left(1^{\text {st }}\right.$ and $\left.17^{\text {th }}\right)(p$ $=.002)$ and treatments pre and post $\left(1^{\text {st }}\right.$ and $\left.35^{\text {th }}\right)(\mathrm{p}=.026)$.

\section{Inflammatory Markers}

Results from the repeated measures ANOVA showed no main effect of time point on CRP and IL-6. There was no significant difference ( $\mathrm{p} \leq 0.05)$ in either variable. There was, however, a slight decrease in CRP and IL-6 values from the $1^{\text {st }}$ to $35^{\text {th }}$ treatments. Although the decrease in inflammation was not significant, this still shows positive effects coming from the pneumatic compression treatment. The slight decrease may also contribute to the significantly lower HbA1C values (Table 3 and 4).

Table 3: Comparison of CRP and IL-6 on Pre, Middle and Post Treatment.

\begin{tabular}{|c|c|c|}
\hline Variable & Mean \pm SD & Effect Size \\
\hline CRP1 & $13.4 \pm 11.6$ & 0.181 \\
\hline CRP2 & $13.0 \pm 8.6$ & \\
\hline CRP3 & $9.9 \pm 6.7$ & \\
\hline IL61 & $3.3 \pm 1.8$ & 0.066 \\
\hline IL62 & $3.4 \pm 2.0$ & \\
\hline IL63 & $3.0 \pm 1.7$ & \\
\hline
\end{tabular}

\begin{tabular}{|c|c|c|c|c|}
\hline Pair & Mean \pm SD & Mean \pm SD & $\Delta$ & Sig. (2-tailed) \\
\hline $\begin{array}{l}\text { PhysicalFunctioning1 - } \\
\text { PhysicalFunctioning2 }\end{array}$ & $\begin{array}{l}\text { PhysicalFunctioning1 } 51.4 \\
\pm 8.2\end{array}$ & $\begin{array}{c}\text { PhysicalFunctioning2 } 52.6 \\
\pm 7.5\end{array}$ & -1.3 & 0.14 \\
\hline $\begin{array}{l}\text { RolePhysical1 - } \\
\text { RolePhysical2 }\end{array}$ & RolePhysical1 $52.4 \pm 7.9$ & RolePhysical2 $54.2 \pm 7.7$ & -1.7 & 0.191 \\
\hline BodilyPlan1 - BodilyPlan2 & BodilyPlan1 $53.2 \pm 8.1$ & BodilyPlan2 $54.0 \pm 6.9$ & -0.8 & 0.644 \\
\hline $\begin{array}{l}\text { GeneralHealth1 - } \\
\text { GeneralHealth2 }\end{array}$ & GeneralHealth1 $45.2 \pm 10.5$ & GeneralHealth2 $47.9 \pm 10.2$ & -2.7 & 0.13 \\
\hline Vitality1 - Vitality2 & Vitality $155.2 \pm 10.5$ & Vitality2 $55.8 \pm 8.4$ & -0.62 & 0.693 \\
\hline $\begin{array}{l}\text { RoleEmotional1 - } \\
\text { RoleEmotional2 }\end{array}$ & RoleEmotional1 $53.5 \pm 5.2$ & RoleEmotional2 $53.5 \pm 6.1$ & 0 & 1 \\
\hline $\begin{array}{l}\text { MentalHealth1 - } \\
\text { MentalHealth2 }\end{array}$ & MentalHealth1 $55.5 \pm 6.1$ & MentalHealth2 $55.4 \pm 5.9$ & 0.09 & 0.923 \\
\hline MCSScore1 - MCSScore2 & MCSScore1 $55.5 \pm 6.1$ & MCSScore2 $55.0 \pm 5.5$ & 0.53 & 0.526 \\
\hline PCSScore1 - PCSScore2 & PCSScore $149.5 \pm 9.1$ & PCSScore2 $51.6 \pm 7.8$ & -2.1 & 0.189 \\
\hline
\end{tabular}

\section{Discussion}

Mean values for CRP decreased from the $1^{\text {st }}$ to the $35^{\text {th }}$ IPC treatment. Although, there was no significant difference in the results for CRP and IL-6, the slight decrease in mean values from these treatments have been shown to have a positive effect on an individual's risk factors for cardiovascular disease and preexisting diabetes. This slight decrease shown in CRP values could lead to a clinical meaningful change in CRP while using this pneumatic compression device. Martin, et al. [10] discussed the positive impact that IPC has on the inflammatory markers (hsCRP, TNF- $\alpha$ ) by the significant difference they found in their results. As a result of their study, both inflammatory markers decreased within the 7 -week treatment therapy. Decreasing these inflammatory markers in individuals that have abnormal glucose tolerance can possibly decrease their risks for cardiovascular diseases and other possible health concerns that may come from them having increased 
inflammation in the blood. Another really important factor among this population is potentially decreasing insulin resistance with the decreased inflammation. Bonetti, et al. [17] studied the effects of EECP on endothelial function in participants with coronary artery disease by using RH-PAT. High inflammation levels can have a negative effect on an individual's endothelium and potentially causing detrimental damage. The endothelium is an important tissue encompassing the blood vessels, heart and lymphatic vessels. There can be negative health risks if this tissue becomes damaged, such as heart failure, stress cardiomyopathy and infections. The results found in this study show that compression therapy may help these individuals benefit from any further cardiovascular complications. The results from these studies show positive outcome from the decreased inflammatory markers, along with the slight decrease in inflammation in the present study that examined CRP and Il-6.

Diabetic neuropathy and IPC treatments were researched by Salek, Bahrpeyma and Mohajeri-Tehrani [18]. They found that there may be some positive effects for individuals dealing with diabetic neuropathy. This could be due to the increased blood flow from the compression treatments that will help the nerve damage caused from hyperglycemia. The increased circulation shown could help individuals with type 2 diabetes suffering from a decrease in blood flow to the lower extremities. There may be some positive outcomes from the use of IPC for diabetics who are suffering from diabetic neuropathy.

Obese and diabetic individuals tend to have increased inflammation levels according to Colwell J [19]. Having these high inflammatory markers can lead to damaging effects of the body. CRP levels have been widely studied as being a potential risk factor for cardiovascular disease. Detrimental vascular injury can be caused from these high inflammatory markers in the blood for an extended period. Seeing a decrease in IL-6 and CRP shows promising results from using the pneumatic compression device. The decreased inflammation levels within the blood can lead to an increased blood flow and possibly more of a decrease in HbA1C. More research needs to be done on the effects of IPC on the diabetic population with the regulation of glucose and inflammation.

The significant decrease found in HbA1c levels from the $1^{\text {st }}$ to $17^{\text {th }}$ and $1^{\text {st }}$ to $35^{\text {th }}$ treatment can lead to many unanswered questions. This significant decrease may be a product of the decrease in inflammation due to the compression therapy. The decrease in inflammatory markers, insulin and glucagon can potentially be a factor to the significantly low HbA1C levels throughout the 7 weeks. The significant decrease in HbA1C levels could have a clinically meaningful change. This significant decrease in this major marker for diabetic individuals could result in potentially controlling glucose better. The IPC treatments may have led to an increase in blood flow shown by Labropolous, et al. [20] that leads to an increase in circulation which can help with lowering inflammation.
The decrease in insulin levels may have something to do with the body using more glucose and becoming less resistant to the insulin being produced. Chronic inflammation can have a negative impact on the body and has been known to have a relation with increased levels of glucose in the blood. If blood flow is increased, due to this compression treatment, then inflammation may be decreased due to recent studies Lawson, et al. [4]. If inflammation is decreased, then this may have a positive effect on $\mathrm{HbA1C}$ levels. More research needs to be done on the effects of chronic inflammation on HbA1C in type 2 diabetics.

Ferrannini, et al. [3] found that high glucagon levels in the body may have a relation with insulin resistance. Glucagon is produced to help blood glucose levels not reach the point of being hypoglycemia. If too much glucagon is found, then there could be a relation to insulin resistance. Furthermore, if glucagon levels in the blood are too high then this may have a correlation to increase blood glucose levels and resistance to insulin in type 2 diabetics that was found with researchers Ferrannini, et al. [3]. The slight decreases found in glucagon and insulin levels could play a part in the significant decrease shown in HbA1C.
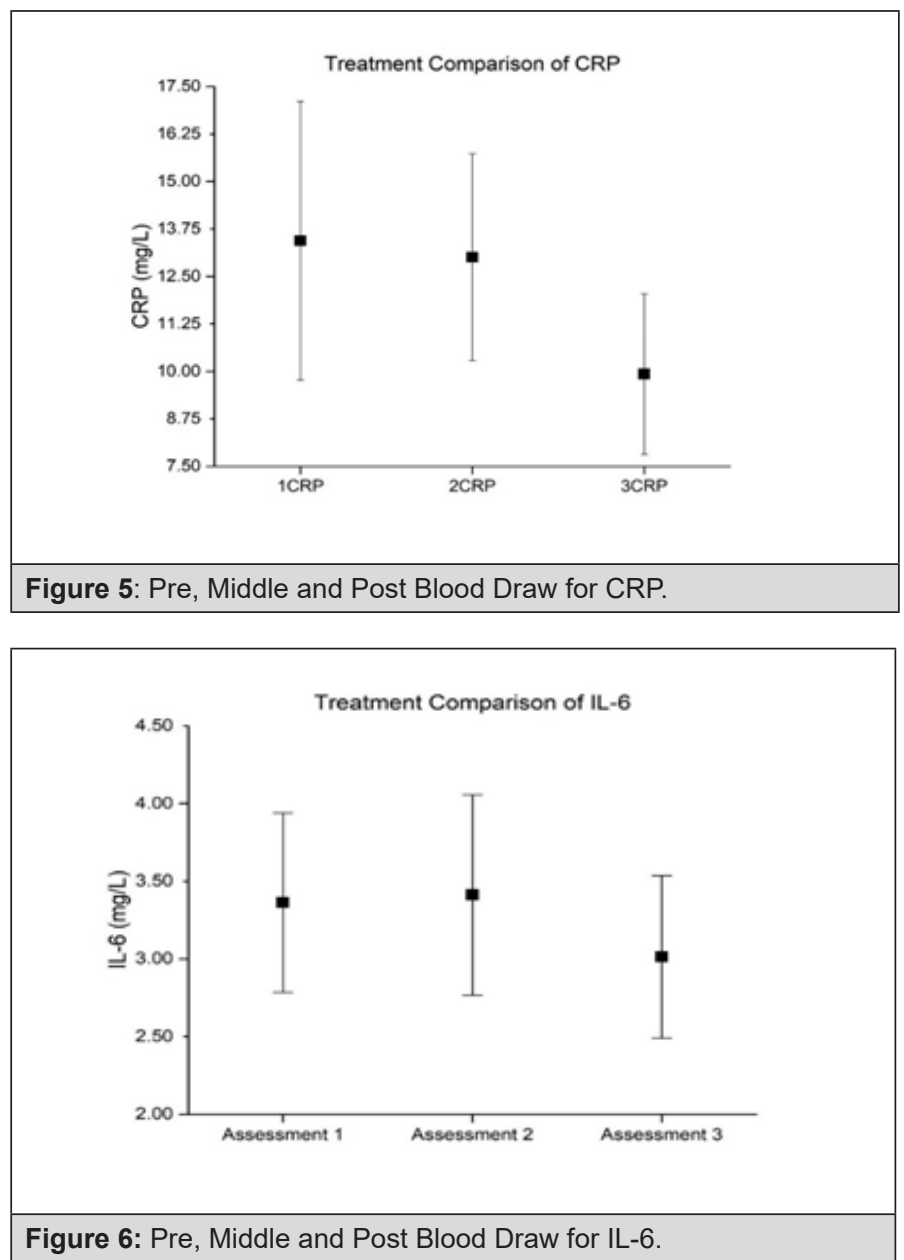

There was no significance found with the SF-36 health survey, but there were some slight increases worth noting. The individuals who participated in the study showed slight increased among their 
general physical health from pre $\left(1^{\mathrm{st}}\right)$ and post $\left(35^{\text {th }}\right)$ treatment surveys. From performing these compression treatments, individuals felt physically better after the $35^{\text {th }}$ treatment when compared to when they first started the treatments. (Figure 5-6).

\section{Acknowledgements}

I would like to thank all of my professors, family and friends for their support throughout this entire process. To Dr. Sparks, Dr. Kullman and Dr. Wadja, thank you all so much for your support, encouragement and guidance. This would not have been possible without the help of you three. I would also like to thank all of my participants for their dedication throughout the study. This would not have been able to happen without your support and participation.

\section{References}

1. Raaijmakers LM, Martens MK, Bagchus C, de Weerdt I, de Vries NK, et al. (2015) Correlates of perceived self-care activities and diabetes control among Dutch type 1 and type 2 diabetics. Journal of Behavioral Medicine 38(3): 450-459.

2. Wilcox G (2005) Insulin and Insulin Resistance. Clinical Biochemist Reviews 26(2): 19-39.

3. Ferrannini E, Muscelli E, Natali A, Gabriel R, Mitrakou A, et al. (2007) Relationship between insulin S, cardiovascular disease risk project I: association of fasting glucagon and proinsulin concentrations with insulin resistance. Diabetologia 50(11): 2342-2347.

4. Lawson WE, Hui JCK, Zheng ZS, Burger L, Jiang L, et al. (1996) Improved Exercise Tolerance following Enhanced External Counterpulsation: Cardiac or Peripheral Effect? Cardiology 87(4): 271-275.

5. Levenson J, Pernollet MG, Iliou MC, Devynck MA, Simon A (2006) Cyclic GMP Release by Acute Enhanced External Counterpulsation. American Journal of Hypertension 19(8): 867-872.

6. Feldman, Arthur M Silver, Marc A Francis, Gary S Abbott smith, Charles W Fleishman, et al. (2006) Enhanced external counter pulsation improves exercise tolerance in patients with chronic heart failure. J Am Coll Cardiol 48(6):1198-1205.

7. Singh-Manoux A, Shipley MJ, Bell JA, Canonico M, Elbaz A, et al. (2017) Association between inflammatory biomarkers and all-cause, cardiovascular and cancer-related mortality. CMAJ 189(10): E384-E390.
8. Haffner SM (2006) The metabolic syndrome, inflammation, diabetes mellitus, and cardiovascular disease. Am J Cardiol 97(2A): 3A-11A.

9. Almstedt HC, Lewis ZH (2016) Intermittent Pneumatic Compression and Bone Mineral Density: An Exploratory Study. J Sport Rehabil 25(1): $1-6$.

10. Martin J, Braith R (2012) Anti-inflammatory effects of enhanced external Counter pulsation in subjects with abnormal glucose tolerance. Appl Physiol Nutr Metab 37(6): 1251-1255.

11. Sutkowska E (2017) Usefulness of intermittent pneumatic compression in medicine. Physiotherapy/Fizjoterapia 24(2): 23-26.

12. Sheldon RD, Roseguini BT, Laughlin MH, Newcomer SC (2013) New insights into the physiologic basis for intermittent pneumatic limb compression as a therapeutic strategy for peripheral artery disease. J Vasc Surg 58(6): 1688-1696.

13. Chen AH, Frangos SG, Kilaru S, Sumpio BE (2001) Intermittent Pneumatic Compression Devices-Physiological Mechanisms of Action. Eur J Vasc Endovasc Surg 21(5): 383-392.

14. Moran PS, Teljeur C, Harrington P, Ryan M (2015) A systematic review of intermittent pneumatic compression for critical limb ischaemia. Vasc Med 20(1): 41-50.

15. Kavros SJ, Delis KT, Turner NS, Voll AE, Liedl DA, et al. (2008) Improving limb salvage in critical ischemia with intermittent pneumatic compression: A controlled study with 18-month follow-up. Journal of Vascular Surgery 47(3): 543-549.

16. Comerota AJ (2011) Intermittent pneumatic compression: Physiologic and clinical basis to improve management of venous leg ulcers. J Vasc Surg 53(4): 1121-1129.

17. Bonetti PO, Barsness GW, Keelan PC, Schnell TI, Pumper GM, et al. (2003) Enhanced external counter pulsation improves endothelial function in patients with symptomatic coronary artery disease. J Am Coll Cardiol 41(10): 1761-1768.

18. Salek S, Bahrpeyma F, Mohajeri-Tehrani M (2015) Intermittent pneumatic compression therapy improves functional and dynamic balance and neuropathy severity in neuropathic patients with type 2 diabetes. International Journal of Diabetes in Developing Countries 35: 439-448.

19. Colwell J (1999) Inflammation and diabetic vascular complications. Diabetes Care. Alexandria 22(12): 1927-1928.

20. Labropoulos N, Leon LR, Bhatti A, Melton S, Kang SS, et al. (2005) Hemodynamic effects of intermittent pneumatic compression in patients with critical limb ischemia. J Vasc Surg (4): 710. 